\title{
Phagocyte-derived catecholamines enhance acute inflammatory injury
}

\author{
Michael A. Flierl ${ }^{1}$, Daniel Rittirsch ${ }^{1}$, Brian A. Nadeau ${ }^{1}$, Anthony J. Chen ${ }^{1}$, J. Vidya Sarma ${ }^{1}$, Firas S. Zetoune ${ }^{1}$, \\ Stephanie R. McGuire ${ }^{1}$, Rachel P. List ${ }^{1}$, Danielle E. Day ${ }^{1}$, L. Marco Hoesel ${ }^{1}$, Hongwei Gao ${ }^{1}$, Nico Van Rooijen ${ }^{3}$, \\ Markus S. Huber-Lang ${ }^{4}$, Richard R. Neubig ${ }^{2}$ \& Peter A. Ward ${ }^{1}$
}

It is becoming increasingly clear that the autonomic nervous system and the immune system demonstrate cross-talk during inflammation by means of sympathetic and parasympathetic pathways $^{1,2}$. We investigated whether phagocytes are capable of de novo production of catecholamines, suggesting an autocrine/paracrine self-regulatory mechanism by catecholamines during inflammation, as has been described for lymphocytes ${ }^{3}$. Here we show that exposure of phagocytes to lipopolysaccharide led to a release of catecholamines and an induction of catecholamine-generating and degrading enzymes, indicating the presence of the complete intracellular machinery for the generation, release and inactivation of catecholamines. To assess the importance of these findings in vivo, we chose two models of acute lung injury. Blockade of $\alpha_{2}$-adrenoreceptors or catecholamine-generating enzymes greatly suppressed lung inflammation, whereas the opposite was the case either for an $\alpha_{2}$-adrenoreceptor agonist or for inhibition of catecholamine-degrading enzymes. We were able to exclude $\mathrm{T}$ cells or sympathetic nerve endings as sources of the injury-modulating catecholamines. Our studies identify phagocytes as a new source of catecholamines, which enhance the inflammatory response.

Although tumor, rubor, dolor and calor (the Latin terms for swelling, redness, pain and heat) are classical features of acute inflammation, the proximal trigger of these responses is neuronal in origin. Similarly, the systemic acute-phase response to infection involves neurone-dependent responses (fever and activation of the hormonal stress response), which are regulated mainly by the hypothalamus ${ }^{2}$. Innate immunity and inflammation are the first lines of defence $^{4}$, with initial responses involving non-specific cellular and humoral pathways, resulting in mediator release ${ }^{1}$. Immune and proinflammatory mediators that are subsequently released rapidly activate neuronal responses that amplify local immune and inflammatory responses designed to contain pathogens and trigger systemic neuroendocrine and regional neural responses that seek to return the system to a homeostatic state ${ }^{2}$. One of the key pathways involved in the neuroendocrine-immune modulating network is the autonomic nervous system. Recently, vagal parasympathetic signalling has been shown to have an important regulatory function in inflammation through cholinergic receptors on phagocytic cells $s^{1,5,6}$. Functional interplay of the adrenergic nervous system with the immune/inflammatory system may counterbalance effects of the parasympathetic nervous system. The presence and synthesis of catecholamines in lymphocytes were first described more than a decade $\operatorname{ago}^{3,7}$. Catecholamines can thereby modulate the proliferation, differentiation and apoptosis of lymphocytes and the production of cytokines through adrenoreceptors expressed on $\mathrm{T}$ and $\mathrm{B}$ cells ${ }^{8-12}$. To investigate whether macrophages and neutrophils (polymorphonuclear cells; PMNs) might be able to generate and release catecholamines, as has been suggested ${ }^{13,14}$, cells were incubated with bacterial lipopolysaccharide (LPS) and levels of noradrenaline and adrenaline in cell supernatants were determined. As shown in Fig. 1a-d, noradrenaline and adrenaline levels increased significantly in cell supernatants 15 min after exposure of macrophages and PMNs to LPS, decreasing at $1 \mathrm{~h}$ and $2 \mathrm{~h}$, and then increasing by $4 \mathrm{~h}$. Experiments were repeated with human PMNs exposed to the potent complement anaphylatoxin $\mathrm{C} 5 \mathrm{a}(10 \mathrm{nM})$, with virtually identical results (data not shown). On the basis of these findings, we conducted experiments to evaluate the presence of two key enzymes involved in catecholamine synthesis in macrophages and PMNs after stimulation with LPS. Tyrosine hydroxylase (TH) is known to be the rate-limiting step in catecholamine synthesis, whereas dopamine $\beta$-hydroxylase (DBH) accounts for the final step of noradrenaline synthesis, converting dopamine to noradrenaline ${ }^{15}$. Macrophages or blood PMNs were incubated for $15 \mathrm{~min}$ and $4 \mathrm{~h}$ with $30 \mathrm{ng} \mathrm{ml}^{-1}$ LPS. Thereafter, messenger RNA expression for catecholamine-generating enzymes was analysed by real-time polymerase chain reaction (PCR). Low baseline mRNA levels for both key enzymes (TH and $\mathrm{DBH}$ ) were detected in unstimulated macrophages. At $4 \mathrm{~h}$ after cell exposure to LPS, mRNAs for both enzymes were significantly upregulated (Fig. 1e, f). PMNs expressed low constitutive levels of mRNA for TH and $\mathrm{DBH}$, but both were clearly upregulated $4 \mathrm{~h}$ after the addition of LPS (Fig. 1g, h). The actions of catecholamines can be terminated in three ways: by reuptake into nerve terminals, by diffusion into extracellular fluids or by metabolic transformation. Two enzymes are essential in the metabolic inactivation of catecholamines: monoamine oxidase (MAO) and catechol O-methyltransferase (COMT). Unstimulated macrophages contained mRNA for both COMT and MAO-A (Fig. 2a, b). After exposure of cells to LPS, the quantity of mRNA for COMT decreased whereas that for MAO-A increased. In accordance with this, when proteins were analysed by western blotting, exposure to LPS caused a decrease in macrophage COMT (Fig. 2c), whereas the same intervention brought about higher levels of MAO-A protein (Fig. 2d). Similar patterns were found in PMNs. Incubation with LPS decreased the level of mRNA for COMT (Fig. 2e) and increased that of mRNA for MAO-A (Fig. 2f). COMT protein amounts decreased when PMNs were exposed to LPS (Fig. 2g); the opposite was true for MAO-A (Fig. 2h). During COMT-mediated $O$-methylation of catecholamines, $S$-adenosylmethionine (SAM) serves as the methyl donor and is subsequently converted to S-adenosyl-L-homocysteine (SAH) after donation of the methyl group to the substrate. It has been known for many years that elevated SAH levels can serve as a strong inhibitor of the COMT-mediated $O$-methylation metabolism of catechols ${ }^{16,17}$. Increased phagocyte 
production of catecholamines might therefore lead to an increased turnover of COMT, increasing levels of the powerful feedback inhibitor SAH and ultimately causing the downregulation of cellular mRNA and protein levels of COMT (Fig. 2). Collectively, these data indicate that LPS-stimulated macrophages and PMNs express catecholamine-generating and degrading enzymes and release noradrenaline and adrenaline after exposure to LPS in vitro. Thus, phagocytes possess the complete cellular machinery to generate catecholamines de novo and to release and inactivate them.

To evaluate the relevance of these findings in vivo, we used two different models of acute lung injury (induced by LPS and by IgG immune complex) to study the effects of adrenoceptor blockade in the setting of a single-organ injury. Adrenoceptors were blocked
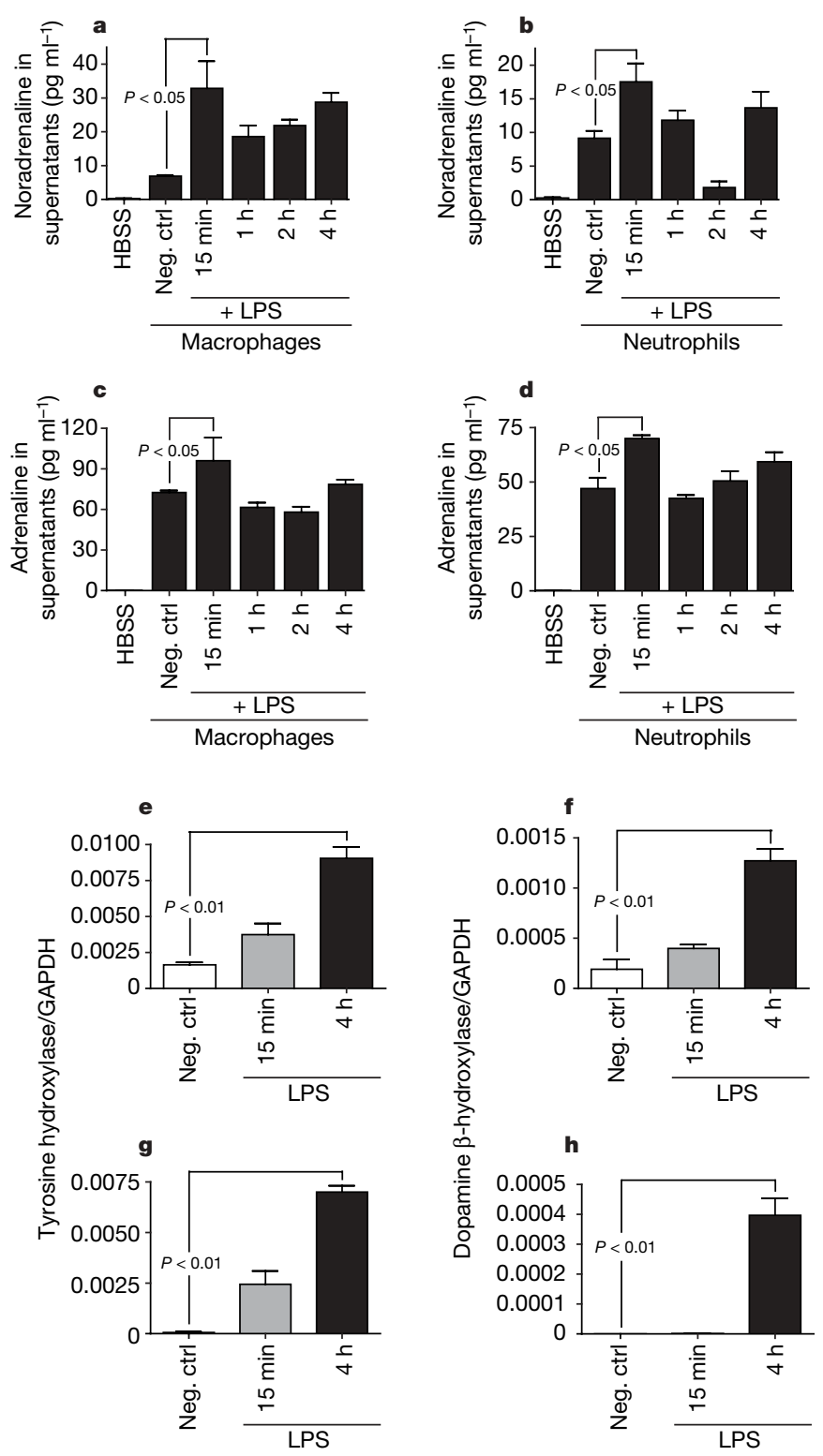

Figure 1 | Release of catecholamines from phagocytes and the presence of catecholamine-producing enzymes in phagocytes. a-d, After isolation, alveolar macrophages and blood PMNs were stimulated with $30 \mathrm{ng} \mathrm{ml}^{-1}$ of LPS in vitro. Cellular supernatant fluids were collected as a function of time thereafter and then analysed by enzyme-linked immunosorbent assay for noradrenaline $(\mathbf{a}, \mathbf{b})$ and adrenaline $(\mathbf{c}, \mathbf{d})$. HBSS served as negative control (neg. ctrl).e-h, After stimulation with $30 \mathrm{ng} \mathrm{ml}^{-1}$ of LPS, mRNA from alveolar macrophages $(\mathbf{e}, \mathbf{f})$ and neutrophils $(\mathbf{g}, \mathbf{h})$ was isolated and subjected to realtime PCR analysis for tyrosine hydroxylase and dopamine $\beta$-hydroxylase. Each bar represents $n \geq 6$ samples. All data are presented as means \pm s.e.m. in vivo by using the appropriate antagonists in the two different models of lung inflammatory injury (Fig. 3a, c). The antagonists were delivered intraperitoneally. Vascular leakage of albumin into lungs, which correlates with lung injury, was measured after the deposition of IgG immune complexes (IC) or LPS in the lung. $\alpha_{1}$-Adrenoceptors were blocked by prazosin, whereas $\alpha_{2}$-adrenoceptors were blocked with RX 821002. $\beta_{1}$-Adrenoceptors were blocked by metoprolol, and $\beta_{2}$-adrenoceptor blockade was induced by ICI 118551 . Only blockade of the $\alpha_{2}$-adrenoceptors significantly reduced the intensity of the albumin leak into lung in the two models of injury, whereas blockade of other receptors neither intensified nor reduced the level of lung injury (Fig. 3a, c). Blockade of the $\beta_{2}$-adrenoceptors with different doses of ICI $118551\left(10-20 \mathrm{mg} \mathrm{kg}^{-1}\right)$ failed to alter injury intensity significantly (data not shown). To clarify the significance of the $\alpha_{2}$-adrenoceptors in our models of lung injury, an $\alpha_{2}$-adrenergic agonist (UK 14304) was used to assess effects on lung injury. As shown in Fig. 3b, d, the severity of lung damage increased twofold and fourfold, respectively, when induction of IgG or LPS lung injury was followed immediately by intraperitoneal administration of UK 14304 , whereas the $\alpha_{2}$-adrenoceptor inhibitor RX 821002 substantially suppressed lung injury in each of the two models. The effects of these interventions were consistent with the morphological evaluation of lungs (Fig. 3e-k). Although the levels of lung injury with LPS and IC (positive controls) were comparable (Fig. 3f, i, respectively), animals treated with UK 14304 showed clearly increased evidence of injury as reflected in increased PMN numbers, fibrin and haemorrhage in the interstitial and alveolar compartments (Fig. $3 g$, j). In sharp contrast, blockade of the $\alpha_{2}$-adrenoceptor by RX 821002 reduced the intensity of inflammation and injury (Fig. 4h, k) in comparison with positive controls, indicating a key function for the a
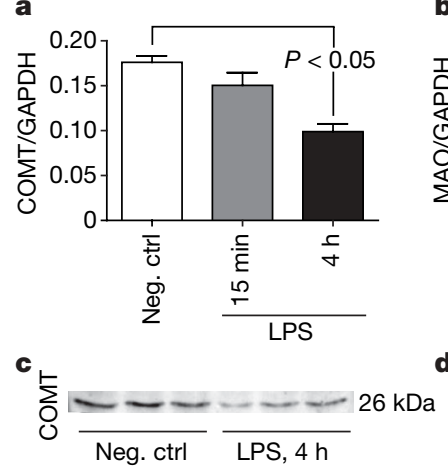

e

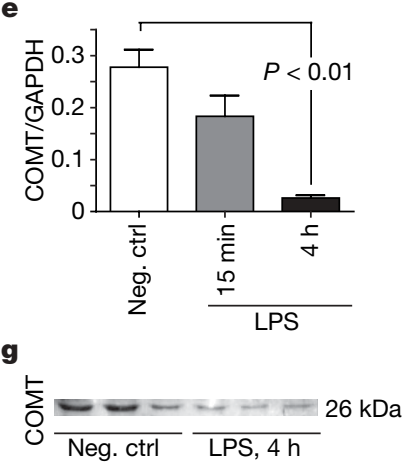

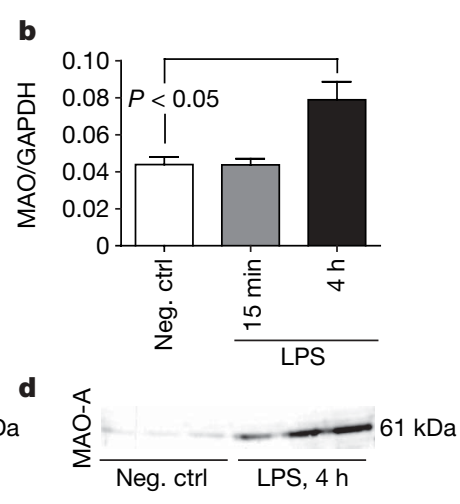

f

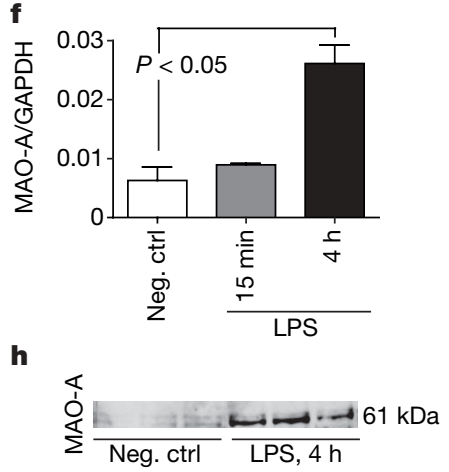

Figure 2 | Expression of catecholamine-inactivating enzymes in phagocytes. After isolation of macrophages and PMNs and stimulation in vitro with $30 \mathrm{ng}$ of LPS, mRNA and protein from alveolar macrophages (a-d) and blood PMNs (e-h) were subjected to analysis by real-time PCR $(\mathbf{a}, \mathbf{b}, \mathbf{e}, \mathbf{f})$ and western blotting $(\mathbf{c}, \mathbf{d}, \mathbf{g}, \mathbf{h})$ for the catecholamine-inactivating enzymes COMT (a, c, e, g) or monoamine oxidase A (MAO-A)

$(\mathbf{b}, \mathbf{d}, \mathbf{f}, \mathbf{h})$. Western blots were repeated at least three separate times.

Representative blots are shown. Real-time PCR data are presented as means \pm s.e.m.; $n \geq 5$ per bar. Neg. ctrl, negative control. 
$\alpha_{2}$-adrenoceptor in these models of lung injury. $\alpha_{2}$-Adrenoceptors belong to the G-protein-coupled, seven-transmembrane receptor family ${ }^{18}$ and are currently divided into three subtypes: $\alpha_{2 \mathrm{~A}}, \alpha_{2 \mathrm{~B}}$ and $\alpha_{2 C}$ (ref. 19). In the present study we found that both alveolar macrophages and blood PMNs express all three subtypes of the $\alpha_{2}$ adrenoceptors (Supplementary Fig. 1).

To exclude T cells or the sympathetic nerve endings as sources of the injury-modulating catecholamines, we achieved T-cell depletion with an antibody and sympathetic denervation by administering reserpine. Recovery of sympathetic function after administration of reserpine takes up to several weeks ${ }^{20}$. In the present study we harvested and exposed PMNs and alveolar macrophages to LPS 4 days after administration of reserpine in vivo to determine whether the production of catecholamines by phagocytes is impaired by reserpine. There was no difference in noradrenaline production between phagocytes from control and reserpine-treated animals (Fig. 4a). Accordingly, we induced lung injury in the presence or absence of $\alpha_{2}$-adrenoceptor blockade in control animals and animals treated 4 days earlier with reserpine. Injury levels were significantly increased in reserpine-treated animals, whereas $\alpha_{2}$-adrenoceptor blockade completely abolished vascular leakage (Fig. 4c). We repeated these experiments in vitro and in vivo $2 \mathrm{~h}$ after reserpine administration, with identical results (data not shown). As expected, adrenal gland homogenates from reserpine-treated animals had almost no detectable noradrenaline (Fig. 4b). In parallel experiments, mice were depleted of CD4 $\mathrm{T}$ cells and the intensity of albumin leakage was assessed. There was no change in the level of injury between T-cell-depleted and isotype-control-treated littermates. In the same animals, $\alpha_{2}$-adrenoceptor blockade was protective (Fig. $4 \mathrm{~d}$ ). Bronchoalveolar lavage (BAL) fluid analysis was performed to determine whether the sympathetic nervous system and T cells were key sources of catecholamines in current models of lung injury. Peak levels of adrenaline occurred in BAL fluids $2 \mathrm{~h}$ after the initiation of immune-complex-mediated injury (Fig. 4e). In parallel, an adrenaline peak was found $3 \mathrm{~h}$ after the initiation of LPS-induced lung injury (data not shown). Other mice were depleted of PMNs, alveolar macrophages or $\mathrm{T}$ cells or were pharmacologically sympathectomized and subjected to lung injury. BAL fluids were obtained and measured $2 \mathrm{~h}$ after the initiation of injury. Whereas depletion of PMNs and alveolar macrophages significantly decreased the presence
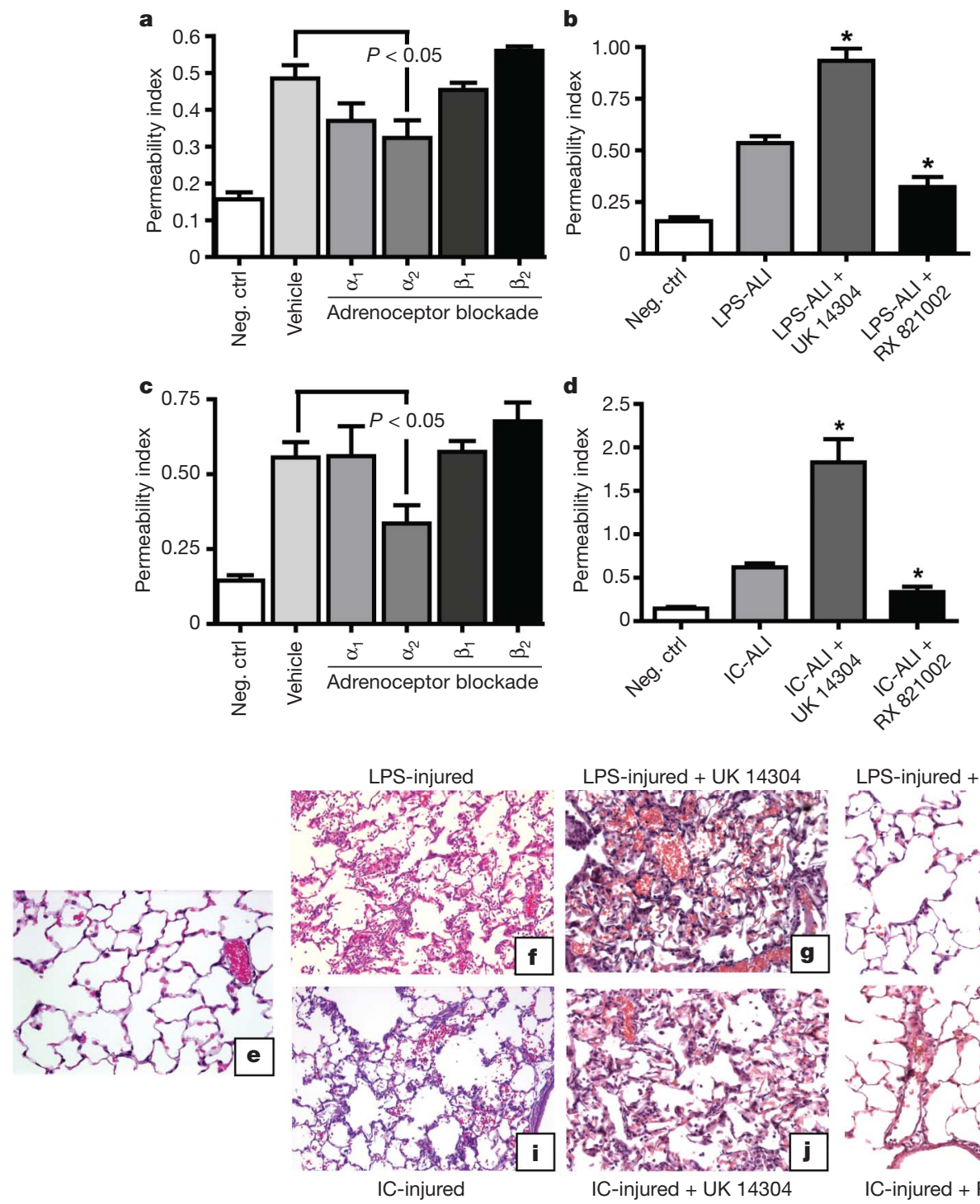

Figure 3 | The $\alpha_{2}$-adrenoceptor mediates the severity of experimental acute lung injury. $\mathbf{a}-\mathbf{d}$, Acute lung injury (ALI) was induced by LPS $(\mathbf{a}, \mathbf{b})$ or IgG IC (c, d) in rats and injury severity was assessed by vascular leak of vascular ${ }^{125} \mathrm{I}$-albumin. Adrenoceptors were blocked pharmacologically as described in the text. All data are presented as means \pm s.e.m.; $n \geq 5$ per bar.

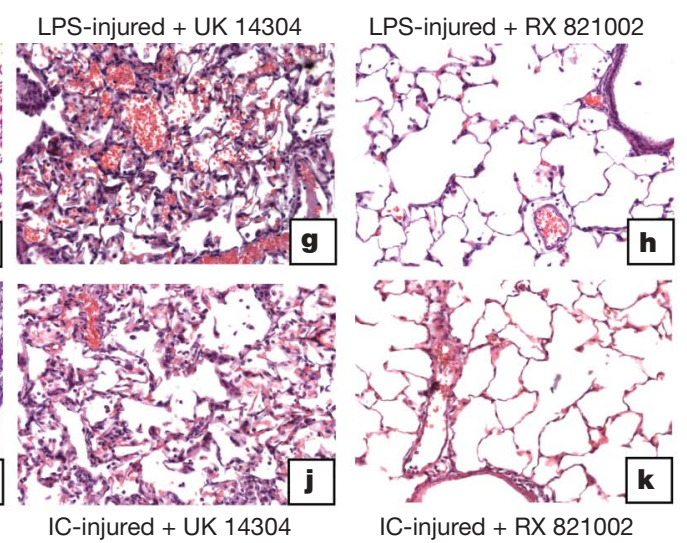

Asterisks in $\mathbf{b}$ and $\mathbf{d}$ indicate $P<0.01$ versus LPS-induced and IC-induced lung injury, respectively. e-k, Hematoxylin and eosin-stained lung sections. e, Uninjured lung. $\mathbf{f}-\mathbf{h}$, LPS-injured animals. $\mathbf{i}-\mathbf{k}$, IC-injured littermates.

Sections are representative for $n \geq 3$ per group. Neg. ctrl, negative control. 

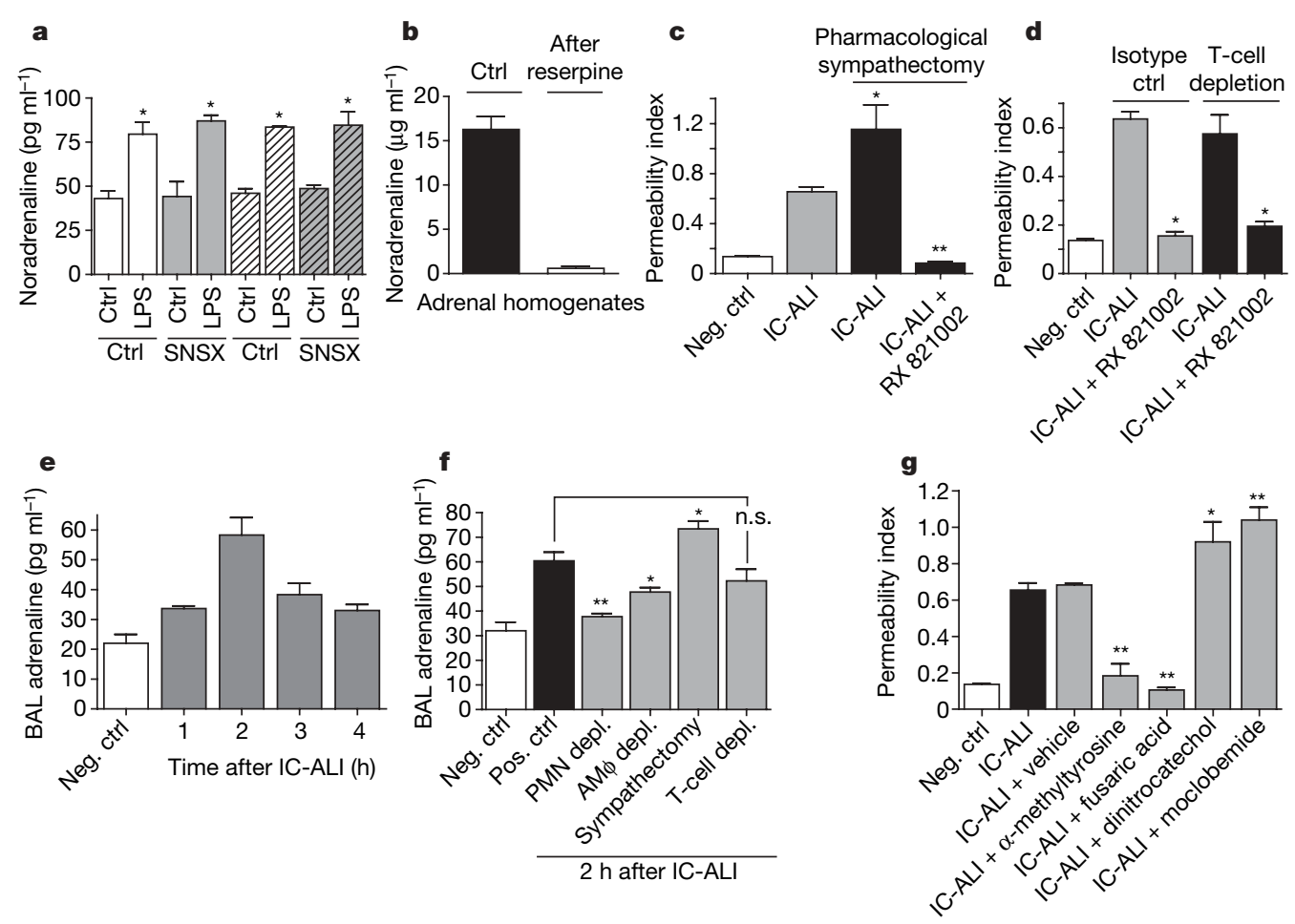

Figure 4 | Phagocyte-derived catecholamines mediate lung injury. a, After sympathectomy (SNSX), noradrenaline production in supernatants of LPSstimulated phagocytes was assessed. Open bars, PMNs; hatched bars, alveolar macrophages. Asterisk, $P<0.01$ versus respective control (ctrl). b, Sympathectomy was confirmed by adrenal gland homogenates. c, Severity of IC-induced acute lung injury (IC-ALI) in sympathectomized animals. Asterisk, $P<0.05$ versus IC-ALI; two asterisks, $P<0.01$ versus IC-ALI. d, Mice were T-cell depleted, and lung injury was compared with that in isotype-antibody-treated littermates. Asterisk, $P<0.01$ versus IC-ALI.

of adrenaline in BAL fluids, reserpine-induced sympathectomy did not decrease adrenaline levels (Fig. 4f). These data indicate that the presence of adrenaline in BAL fluids requires PMNs and macrophages but not $\mathrm{T}$ cells. Phagocytes of adrenalectomized animals have higher baseline levels of TH, DBH and catecholamine secretion (M.A.F., D.R., R.P.L., A.J.C., D.E.D., B.A.N., F.S.Z., J.V.S., A. B. Lentsch, M.S.H.-L. and P.A.W., unpublished observations). It might be possible that the baseline production of catecholamines in phagocytes is increased as a compensatory endogenous mechanism to pharmacological sympathectomy. Last, we showed that pharmacological blockade of the catecholamine-generating TH (by using $\alpha$-methyltyrosine) and DBH (by using fusaric acid) greatly diminished the inflammatory response, whereas inhibition of the catecholamine-degrading enzymes COMT (with dinitrocatechol) and MAO (with moclobemide) significantly increased lung injury as defined by the permeability index (Fig. 4g).

Alveolar macrophages and PMNs have a central function in the pathophysiology of IgG IC-induced lung injury ${ }^{21}$. The $\alpha_{2}$ adrenoreceptor is expressed on alveolar macrophages ${ }^{11}$, and modulation of the $\alpha_{2}$-adrenoceptor regulates LPS-induced production of tumour necrosis factor (TNF)- $\alpha$ in vitro from alveolar macrophages $^{22}$. We sought to determine whether in vitro blockade of the adrenoceptors, especially the $\alpha_{2}$-adrenoreceptor, might be linked to the present findings in vivo. There was a variable inhibition in vitro of various macrophage-derived cytokines (TNF- $\alpha$, interleukin- 6 and interleukin-1 $\beta$ ) and chemokines (cytokine-induced neutrophil chemoattractant (CINC)-1) by $\alpha_{1}$-adrenoceptor, $\alpha_{2}$-adrenoceptor, $\beta_{1}$-adrenoceptor or $\beta_{2}$-adrenoceptor blockade (Supplementary Table 1), with blockade of the $\alpha_{2}$-adrenoreceptor achieving the most consistent suppression of mediators. Identical experiments with PMNs revealed similar patterns (data not shown). e, Adrenaline peak was determined in BAL fluids. f, Mice were depleted (depl.) of PMN, alveolar macrophages (AM $\Phi)$ or T cells or were sympathectomized, and adrenaline was measured $2 \mathrm{~h}$ after IC-ALI. Asterisk, $P<0.05$ versus positive control (pos. ctrl); two asterisks, $P<0.01$ versus positive control. Neg. ctrl, negative control. g, Severity of lung injury after inhibition of TH, DBH, COMT or MAO. Asterisk, $P<0.05$ versus IC-ALI plus vehicle; two asterisks, $P<0.01$ versus IC-ALI plus vehicle. Data are presented as means \pm s.e.m.; $n \geq 5$ per bar.

Taken together, the results of this study indicate that both alveolar macrophages and blood PMNs are capable of the production of catecholamines de novo and of their release, implicating phagocytes as a previously unrecognized source of catecholamines. Enhanced production or decreased generation of catecholamines (with the use of inhibitors of catecholamine-generating or catecholaminedegrading enzymes) increases or decreases the intensity of inflammatory injury, respectively. Blockade of $\alpha_{2}$-adrenoceptors in vivo greatly decreases the intensity of inflammatory injury. In many respects, the phagocytic system can be looked on as a diffusely expressed adrenergic organ.

\section{METHODS SUMMARY}

Animals and anaesthesia. All studies were conducted in accordance with the University of Michigan Committee on Use and Care of Animals. Specific pathogen-free male Long-Evans rats (300-325 g; Taconic) or C57BL/6 mice (Jackson Laboratory) were anaesthetized with isoflurane (Aerrane; Henry Schein).

Isolation of PMNs and alveolar macrophages. Whole blood was drawn into syringes containing anticoagulant citrate dextrose (ACD) (Baxter Health Care), in a volume ratio of 1:10. Neutrophils were isolated by using Ficoll-Paque gradient centrifugation (Pharmacia Biotech $\mathrm{AB}$ ) followed by a dextran sedimentation step. After hypotonic lysis of residual red blood cells, neutrophils were resuspended in Hanks balanced salt solution (HBSS) containing $\mathrm{Ca}^{2+}$ and $\mathrm{Mg}^{2+}$. Rat alveolar macrophages were isolated by repeatedly lavaging the lungs of anaesthetized healthy animals. After centrifugation of BAL fluids, cells were resuspended in HBSS containing $\mathrm{Ca}^{2+}$ and $\mathrm{Mg}^{2+}$.

Stimulation of phagocytes by LPS. Macrophages or PMNs $\left(3 \times 10^{6}\right.$ or $5 \times 10^{6}$ in $1.0 \mathrm{ml}$ ) were isolated and incubated with 30 or $50 \mathrm{ng}$ of LPS (Escherichia coli 0111:B4; Sigma Aldrich). Supernatants were obtained and frozen at $-80^{\circ} \mathrm{C}$. Enzyme-linked immunosorbent assay/enzyme immunoassay analysis of cell supernatants. Noradrenaline and adrenaline enzyme immunoassays were obtained from Rocky Mountain Diagnostics. 
Alveolitis induced by IgG IC and by LPS. IgG IC-induced alveolitis was performed as described previously ${ }^{23,24}$. For LPS-induced alveolitis, rats received $300 \mu \mathrm{g}$ of LPS (Sigma Aldrich) intratracheally.

Morphological assessment of lung injury. At $4 \mathrm{~h}$ after IgG IC deposition or $6 \mathrm{~h}$ after LPS deposition, lungs were fixed by intratracheal instillation of $10 \mathrm{ml}$ of PBS-buffered ( $\mathrm{pH}$ 7.2) formalin (10\%). The lungs were further fixed in a $10 \%$ buffered formalin solution for histological examination by tissue sectioning and staining with haematoxylin and eosin.

Statistical analysis. All values are expressed as means \pm s.e.m. Data were analysed with ANOVA and a Student-Newman-Keuls test. Differences were considered significant at $P \leq 0.05$.

Full Methods and any associated references are available in the online version of the paper at www.nature.com/nature.

Received 19 July; accepted 20 August 2007.

Published online 30 September 2007.

1. Tracey, K. J. The inflammatory reflex. Nature 420, 853-859 (2002)

2. Sternberg, E. M. Neural regulation of innate immunity: a coordinated nonspecific host response to pathogens. Nature Rev. Immunol. 6, 318-328 (2006).

3. Bergquist, J., Tarkowski, A., Ekman, R. \& Ewing, A. Discovery of endogenous catecholamines in lymphocytes and evidence for catecholamine regulation of lymphocyte function via an autocrine loop. Proc. Natl Acad. Sci. USA 91, 12912-12916 (1994)

4. Cook, D. N., Pisetsky, D. S. \& Schwartz, D. A. Toll-like receptors in the pathogenesis of human disease. Nature Immunol. 5, 975-979 (2004).

5. Borovikova, L. V. et al. Vagus nerve stimulation attenuates the systemic inflammatory response to endotoxin. Nature 405, 458-462 (2000).

6. Wang, H. et al. Nicotinic acetylcholine receptor $\alpha 7$ subunit is an essential regulator of inflammation. Nature 421, 384-388 (2003).

7. Musso, N. R., Brenci, S., Setti, M., Indiveri, F. \& Lotti, G. Catecholamine content and in vitro catecholamine synthesis in peripheral human lymphocytes. J. Clin. Endocrinol. Metab. 81, 3553-3557 (1996).

8. Swanson, M. A., Lee, W. T. \& Sanders, V. M. IFN- $\gamma$ production by Th1 cells generated from naive $\mathrm{CD} 4^{+}$T cells exposed to norepinephrine. J. Immunol. 166, 232-240 (2001).

9. Sanders, V. M. et al. Differential expression of the $\beta_{2}$-adrenergic receptor by Th and Th2 clones: implications for cytokine production and B cell help. J. Immunol. $158,4200-4210$ (1997)

10. Kohm, A. P., Mozaffarian, A. \& Sanders, V. M. B cell receptor- and $\beta_{2}$-adrenergic receptor-induced regulation of B7-2 (CD86) expression in B cells. J. Immunol. 168, 6314-6322 (2002)

11. Bergquist, J., Ohlsson, B. \& Tarkowski, A. Nuclear factor-кB is involved in the catecholaminergic suppression of immunocompetent cells. Ann. NY Acad. Sci. 917, 281-289 (2000).
12. Torres, K. C. et al. Norepinephrine, dopamine and dexamethasone modulate discrete leukocyte subpopulations and cytokine profiles from human PBMC. J. Neuroimmunol. 166, 144-157 (2005).

13. Brown, S. W. et al. Catecholamines in a macrophage cell line. J. Neuroimmunol. 135, 47-55 (2003).

14. Cosentino, M. et al. Endogenous catecholamine synthesis, metabolism, storage and uptake in human neutrophils. Life Sci. 64, 975-981 (1999).

15. Elenkov, I. J., Wilder, R. L., Chrousos, G. P. \& Vizi, E. S. The sympathetic nerve-an integrative interface between two supersystems: the brain and the immune system. Pharmacol. Rev. 52, 595-638 (2000).

16. Ueland, P. M. Pharmacological and biochemical aspects of S-adenosylhomocysteine and S-adenosylhomocysteine hydrolase. Pharmacol. Rev. 34, 223-253 (1982)

17. Zhu, B. T. Catechol-O-methyltransferase (COMT)-mediated methylation metabolism of endogenous bioactive catechols and modulation by endobiotics and xenobiotics: importance in pathophysiology and pathogenesis. Curr. Drug Metab. 3, 321-349 (2002).

18. Bone, R. C., Francis, P. B. \& Pierce, A. K. Intravascular coagulation associated with the adult respiratory distress syndrome. Am. J. Med. 61, 585-589 (1976).

19. Bylund, D. B. et al. International Union of Pharmacology nomenclature of adrenoceptors. Pharmacol. Rev. 46, 121-136 (1994).

20. Hoffman, B. B. in Goodman \& Gilman's The Pharmacological Basics of Therapeutics (eds Brunton, L. L., Lazo, J. S. \& Parker, K. L.) 845-868 (McGraw-Hill, New York, 2006).

21. Ward, P. A. Rous-Whipple Award Lecture. Role of complement in lung inflammatory injury. Am. J. Pathol. 149, 1081-1086 (1996).

22. Spengler, R. N., Allen, R. M., Remick, D. G., Strieter, R. M. \& Kunkel, S. L. Stimulation of $\alpha$-adrenergic receptor augments the production of macrophage-derived tumor necrosis factor. J. Immunol. 145, 1430-1434 (1990)

23. Czermak, B. J. et al. Synergistic enhancement of chemokine generation and lung injury by $\mathrm{C} 5 \mathrm{a}$ or the membrane attack complex of complement. Am. J. Pathol. 154, 1513-1524 (1999).

24. Huber-Lang, M. et al. Generation of $\mathrm{C} 5 \mathrm{a}$ in the absence of C3: a new complement activation pathway. Nature Med. 12, 682-687 (2006).

Supplementary Information is linked to the online version of the paper at www.nature.com/nature.

Acknowledgements We thank B. Schumann and S. Scott for assistance in the preparation of this manuscript. This study was supported by NIH grants to P.A.W. and R.R.N., and by Deutsche Forschungsgemeinschaft grants to M.S.H.-L.

Author Information Reprints and permissions information is available at www.nature.com/reprints. Correspondence and requests for materials should be addressed to P.W. (pward@umich.edu). 


\section{METHODS}

Western blot analysis of MAO-A, COMT, $\alpha_{2 \mathrm{~A}}$-adrenoceptors, $\alpha_{2 \mathrm{~B}}$

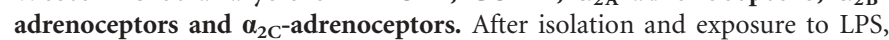
phagocytes were lysed with Laemmli buffer containing 5\% mercaptoethanol. Samples were then separated in a denaturing polyacrylamide gel and transferred to a nitrocellulose membrane. After blocking (5\% Tris-buffered saline pH 7.0 containing $0.1 \%$ Tween 20 ) and washing, membranes were then incubated overnight with primary antibodies against adrenoreceptors, MAO-A and COMT (Santa Cruz Biotechnology) and antibodies against GAPDH (Abcam) at $4{ }^{\circ} \mathrm{C}$. Membranes were analysed by enhanced chemiluminescence.

Isolation of mRNA and detection of rat $\alpha_{2 \mathrm{~A}}$-adrenoreceptors, $\alpha_{2 \mathrm{~B}}$ adrenoreceptors, $\alpha_{2 \mathrm{C}}$-adrenoreceptors, TH, DBH, MAO-A and COMT by quantitative real-time PCR analysis. Total RNA was extracted from phagocytes with Trizol reagent (Life Technologies) in accordance with the manufacturer's instructions. Reverse transcription was performed with $1 \mu \mathrm{g}$ of RNA by using AMV Reverse Transcriptase (Roche). Real-time quantitative PCR was then performed with SYBR Green PCR Master Mix (Applied Biosystems). The following primer pairs were used: rat $\alpha_{2 \mathrm{~A}}$ adrenoreceptor 5 '-CTT GGC CCT CGA CGT GCT CTT TTG-3' (forward) and 5' ${ }^{\prime}$-CGG CGG CGC GGA ACA GG-3' (reverse); rat $\alpha_{2 \mathrm{~B}}$ adrenoreceptor, $5^{\prime}$-TCG GCC ATC ACC TTT CTC ATC CTT-3' (forward) and 5'-GGG CGT CGG GGC GTT GGT C-3' (reverse); rat $\alpha_{2 \mathrm{C}}$ adrenoreceptor, $5^{\prime}$-TGG CTC ATC TCG GCT GTC ATC TCC-3' (forward) and $5^{\prime}$-TGT CCC CCT CGG CAC CCT CTC-3' (reverse); rat TH, 5' -AGT CTG GCC TTC CGC GTG TTT CAA-3' (forward) and 5'-GGG CGC TGG ATA CGA GAG GCA TAG-3' (reverse); rat DBH, 5' -CTG CGA CCC CAA GGA TTA TG$3^{\prime}$ (forward) and 5'-CAG CAC GTG GCG ACA GTA GTT-3' (reverse); rat MAO-A, 5'-GTT GAG CGG CTG ATC CAC TTT GT-3' (forward) and 5'CCG GCC TCT CCA GCT TCA CTC T-3' (reverse); rat COMT, 5'-GAC GCG AAA GGC CAA ATC AT-3' (forward) and 5'-GCT GCT GCT CCC TCT CAC ATA-3' (reverse); rat GAPDH, 5'-CGG CAA GTT CAA CGG CAC AGT CA-3' (forward) and 5'-CTT TCC AGA GGG GCC ATC CAC AG-3' (reverse). Product sizes were 403 base pairs (bp) ( $\alpha_{2 \mathrm{~A}}$-adrenoreceptor), 427 bp ( $\alpha_{2 \mathrm{~B}}$-adrenoreceptor), $409 \mathrm{bp}$ ( $\alpha_{2 \mathrm{C}}$-adrenoreceptor), $458 \mathrm{bp}(\mathrm{TH}), 439 \mathrm{bp}$ (DBH), 451 bp (MAO-A), $435 \mathrm{bp}$ (COMT) and $424 \mathrm{bp}$ (GAPDH).

Drugs. All drugs (from Sigma Aldrich unless stated otherwise) were administered intraperitoneally: propane-1,2-diol (vehicle; $1 \mathrm{ml} \mathrm{kg}^{-1}$ ), Prazosin $\left(2 \mathrm{mg} \mathrm{kg}^{-1}\right)$, RX $821002\left(10 \mathrm{mg} \mathrm{kg}^{-1}\right)$, metoprolol $\left(15 \mathrm{mg} \mathrm{kg}^{-1}\right)$, ICI 118551 $\left(10 \mathrm{mg} \mathrm{kg}^{-1}\right)$ and UK $14304\left(2 \mathrm{mg} \mathrm{kg}^{-1}\right), \alpha$-methyltyrosine $\left(200 \mathrm{mg} \mathrm{kg}^{-1}\right)$, fusaric acid $\left(75 \mathrm{mg} \mathrm{kg}^{-1}\right), 3,5$-dinitrocatechol $\left(50 \mathrm{mg} \mathrm{kg}^{-1}\right)$ and moclobemide (20 $\mathrm{mg} \mathrm{kg}^{-1}$; Biotrend).

Depletion of CD4 T cells, neutrophils and alveolar macrophages. For depletion of CD4 T cells, C57BL/6 mice were treated with $0.2 \mathrm{mg}$ of CD4-specific monoclonal antibody (GK1.5; BD Pharmingen) intraperitoneally $48 \mathrm{~h}$ before induction of lung injury. Alveolar macrophages and neutrophils were depleted as described previously ${ }^{25,26}$.

Pharmacological sympathectomy. To achieve pharmacological sympathectomy, mice were injected intraperitoneally with reserpine $\left(5 \mathrm{mg} \mathrm{kg}^{-1}\right)$. Four days after reserpine injection, acute lung injury was initiated as described above.

25. Gao, H. et al. Stat3 activation in acute lung injury. J. Immunol. 172, 7703-7712 (2004).

26. Hoesel, L. M. et al. Harmful and protective roles of neutrophils in sepsis. Shock 24, 40-47 (2005). 